MRC Centre for Global Infectious Disease Analysis, Department of Infectious Disease Epidemiology, School of Public Health, Imperial College, London, UK

2 Grantham Institute-Climate Change and the Environment, Imperial College, London, UK

3 MRC Unit, London School of Hygiene and Tropical Medicine, Atlantic Boulevard, Fajara, Banjul, the Gambia

4 Centre on Climate Change and Planetary Health, London School of Hygiene and Tropical Medicine, London, UK

5 Department of Fish and Wildlife Conservation, Virginia Polytechnic Institute and State University, Blacksburg, Virginia, 24061 USA

6 Centre for Mathematical Modelling of Infectious Diseases, London School of Hygiene and Tropical Medicine, London, UK

7 Barcelona Institute for Global Health, Barcelona, Spain

8 Department of Public Health and Clinical Medicine, Umeå University, Umeå, Sweden

9 Scientific Assessment Section, European Centre for Disease Prevention and Control, 16973 Solna Sweden

10 Institute for Global Health, University College London, London W1T 4TJ, UK

Correspondence to: K Murray kris.murray@imperial.ac.uk; kris.murray@lshtm.ac.uk Cite this as: BMJ 2020;371:m3086 http://dx.doi.org/10.1136/bmj.m3086 Published: 13 November 2020

CLIMATE CHANGE AND COMMUNICABLE DISEASES

\title{
Tracking infectious diseases in a warming world
}

\section{Using infectious diseases sensitive to climate as indicators of climate change helps stimulate and inform public health responses, write Kris A Murray and colleagues}

Kris A Murray, ${ }^{1,2,3,4}$ Luis E Escobar, ${ }^{5}$ Rachel Lowe, ${ }^{4,6,7}$ Joacim Rocklöv, ${ }^{8}$ Jan C Semenza, ${ }^{9}$ Nick Watts ${ }^{10}$

In one of the first articles published by The BMJ on climate change, Haines in 1991 wrote: "Eight of the hottest 10 years this century have occurred since 1980." Noting the influence of temperature on the life cycles of several vectors, hosts, and pathogens, Haines went on to question the implications of predicted climate change for many infectious diseases. It is discomforting that today, three decades later, circumstances have hardly changed, and that early forecasts have begun to ring true. ${ }^{23}$ Eight of the 10 hottest years on record have now occurred since $2010^{4}$; associations between climate change and the burden, transmission, or distribution of many infectious diseases (principally caused by protozoan, helminth, vectorborne, foodborne, soilborne, and waterborne pathogens) are increasingly being reported ${ }^{5}$; the European Centre for Disease Prevention and Control (ECDC) now ranks climate among the most frequently implicated "drivers" of infectious disease threats ${ }^{6}$; and The World Health Organization now recognises climate change as one of the major health challenges of the 21st century. ${ }^{7}$

\section{In such a rapidly changing world, how can} researchers, health professionals, and policy makers keep track of the risks and intervene accordingly? How can policy options be evaluated, particularly when aiming to achieve globally agreed sustainable development, environmental (including the Paris Agreement), and health management targets? ${ }^{89}$

One emerging strategy is the use of climate change "indicators," which aim to keep track of historical and future predicted trends in key impact areas related to climate change. Such indicators have taken on a range of functions, including quantifying and characterizing exposure, vulnerability, and risk for both populations and health systems, identifying and tracking key impacts on population health, and evaluating changes in adaptive capacity and resilience. ${ }^{10}$ Indicator initiatives explicitly aim to go beyond the fractured and often inconsistent evidence base presented in the primary scientific literature to bring together or generate relevant information in some generally consistent fashion. They also tend to focus more specifically on the analysis of trends through time, often with an emphasis on accessible yet powerful data sharing and visualizations to stimulate action across sectors and track progress towards some predefined targets. Here we illustrate how “climate-sensitive infectious diseases" (CSIDs) are being used as climate change indicators to help stimulate and inform public health responses to climate change.

\section{Climate change and health indicators}

An example of a benchmark for the quantification and comparison of varying health outcomes is the Global Burden of Disease (GBD) programme. ${ }^{11}$ This quantifies death and loss of health and wellbeing from hundreds of diseases and their risk factors, and is used to guide health surveillance and improve global health management policies. However, although the GBD programme estimates the global burden of several CSIDs, it does not capture some important but difficult to define health impacts, including from health inequalities or climate change. $^{12}$

Several climate change indicator initiatives exist that seek to partially fill this gap. Indicator initiatives specifically targeting climate change and health are relatively recent but have been advocated for widely, with key efforts including examples from learned societies, ${ }^{12}$ health authorities (eg, the US Centers for Disease Control and Prevention (CDC) ${ }^{13}$ ), other government agencies (eg, the US Environmental Protection Agency, ${ }^{14}$ the European Environment Agency, and the forthcoming proposal for an EU observatory for climate change and health and the EU Adaptation Strategy planned for $2021^{15}$ ), funders (eg, Wellcome Trust ${ }^{16}$ ), and academic consortiums (eg, the Inter-Sectoral Impact Model Intercomparison Project ${ }^{17}$ and the Lancet Countdown on Health and Climate Change ${ }^{18}$ ). Such indicator initiatives range in scale from local to global.

Where numerous indicator initiatives have tackled some of the more direct impacts of climate change on health or those for which greater volume and quality of data exist (eg, heat-related mortality), few indicators exist for more complex, indirect impact areas such as infectious diseases. For example, the CDC Tracking Network currently reports on flood and heat vulnerability trends but not infectious diseases..$^{13}$

\section{Global trends in climate sensitive infectious diseases}

Current CSID indicators focus primarily on the climatic suitability or population vulnerability components of disease transmission risk, as opposed to case or burden data.

To illustrate, we briefly highlight some of our work as part of the Lancet Countdown on Health and Climate Change, ${ }^{19}$ for which we have developed indicators to: (1) assess spatial and temporal trends in the environmental suitability for CSID transmission (for dengue, malaria, and pathogenic Vibrio bacteria); 
and (2) evaluate the changing basis of population vulnerability to arboviruses (ie, factoring in national characteristics that define their propensity to be adversely affected by infectious disease threats, such as public health measures).

Briefly, indicator analyses for dengue, malaria, and pathogenic Vibrio bacteria show increases in the environmental suitability for disease transmission over past decades. For example, 2017 was the second most suitable year on record for the transmission of dengue virus, with average increases of $7.2 \%$ and $9.8 \%$ in vectorial capacity observed in the past five years compared with a 1950 baseline for the key vectors Aedes aegypti and A albopictus, respectively (fig 1). Despite these increases, country level vulnerability to dengue outbreaks (ie, exposure to mosquitoes after controlling for the presence of disease-relevant public health measures) has decreased globally by $31 \%$ since 2010 , although some regions remain more vulnerable than others and progress has reversed in these regions in recent years (fig 2). The number of suitable months per year for the transmission of malaria (Plasmodium falciparum) in the African highlands has increased by $29.9 \%$ in the past five years compared to a 1950 baseline. By contrast, other regions do not show an increasing trend for malaria, potentially due to some areas (eg, lowlands) becoming too warm or experiencing shifts away from the combinations of temperature, rainfall, and humidity that enhance transmission (fig 3). For waterborne diseases caused by pathogenic Vibrio bacteria, similarly strong increases in the percentage of coastal area suitable for transmission are observed at northern latitudes $\left(40-70^{\circ} \mathrm{N}\right)$ (fig 4 top), in the Baltic Sea (fig 4 middle) and along the north east coast of the United States (fig 4 bottom). The number of days per year suitable for Vibrio in the Baltic reached 107 in 2018 , double the early 1980 s baseline (fig 4 middle).

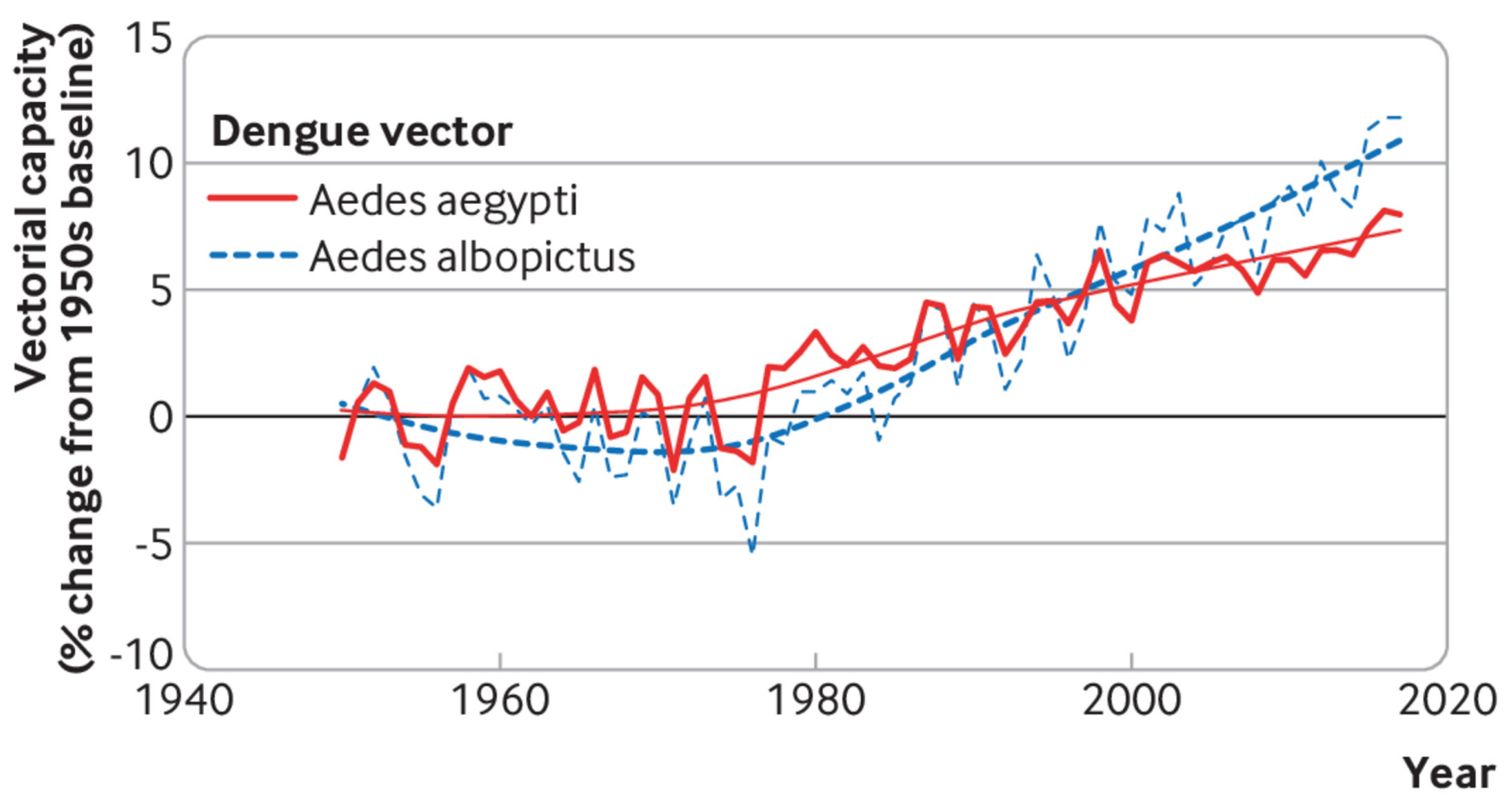

Fig 1 | Mathematical models of dengue vectorial capacity for $A$ aegypti and $A$ albopictus mosquitoes reveal temporal changes in the potential for dengue transmission due to a warming climate since 1950 . 

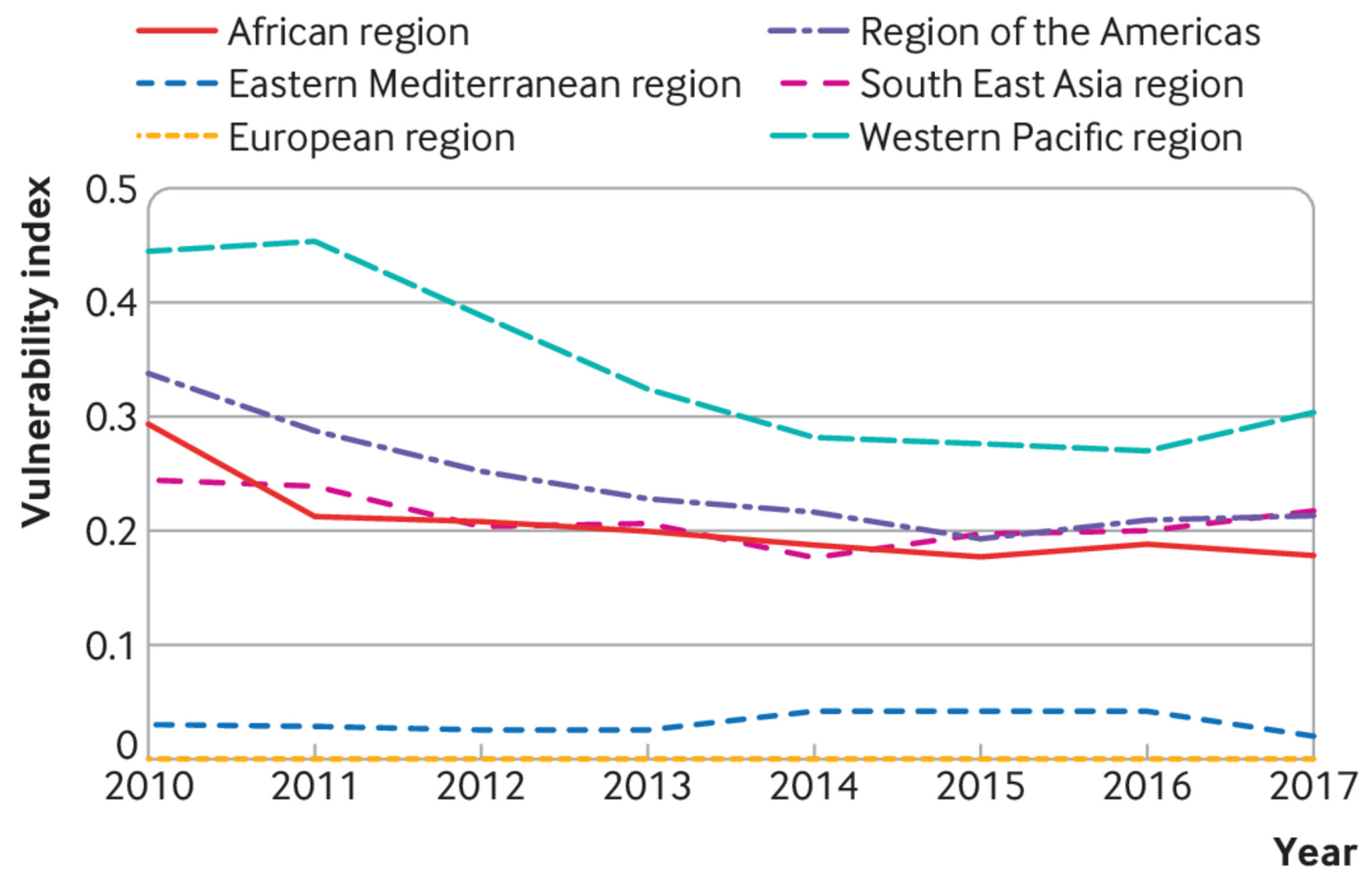

Fig 2 | Despite the increases in environmental suitability for dengue shown in fig 1, improved public health measures have on average lowered vulnerability to dengue outbreaks across most regions since 2010 , although some recent reversals in this trend are observed in the more vulnerable regions. 

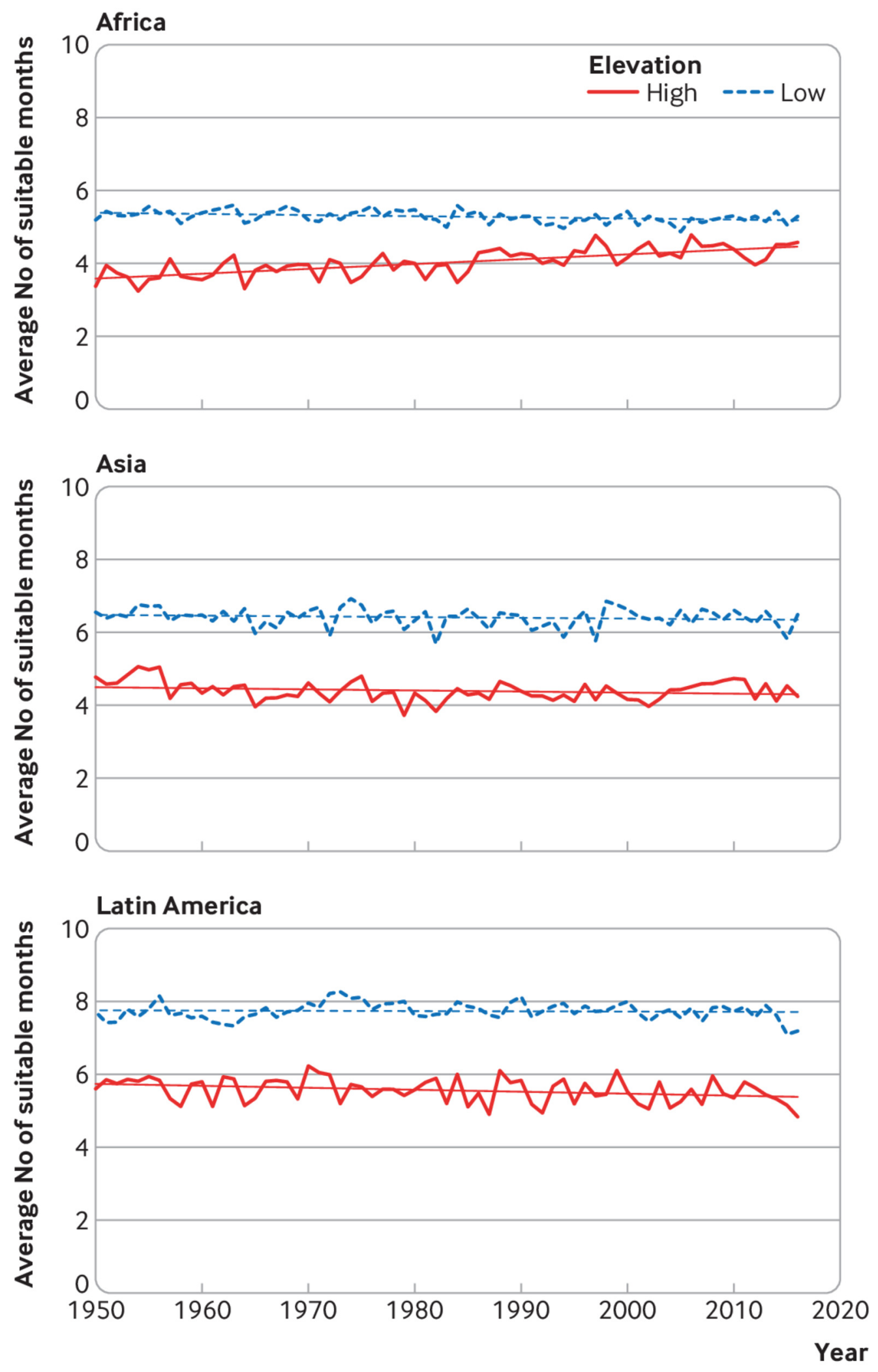

Fig 3 | The number of suitable months a year for malaria transmission during the period 1950-2017, as determined by combinations of temperature, rainfall, and humidity, are increasing predominantly in the African highlands (ie, elevation $>=1500 \mathrm{~m}$ ). No change or subtle declines in environmental suitability are observed in other regions. 

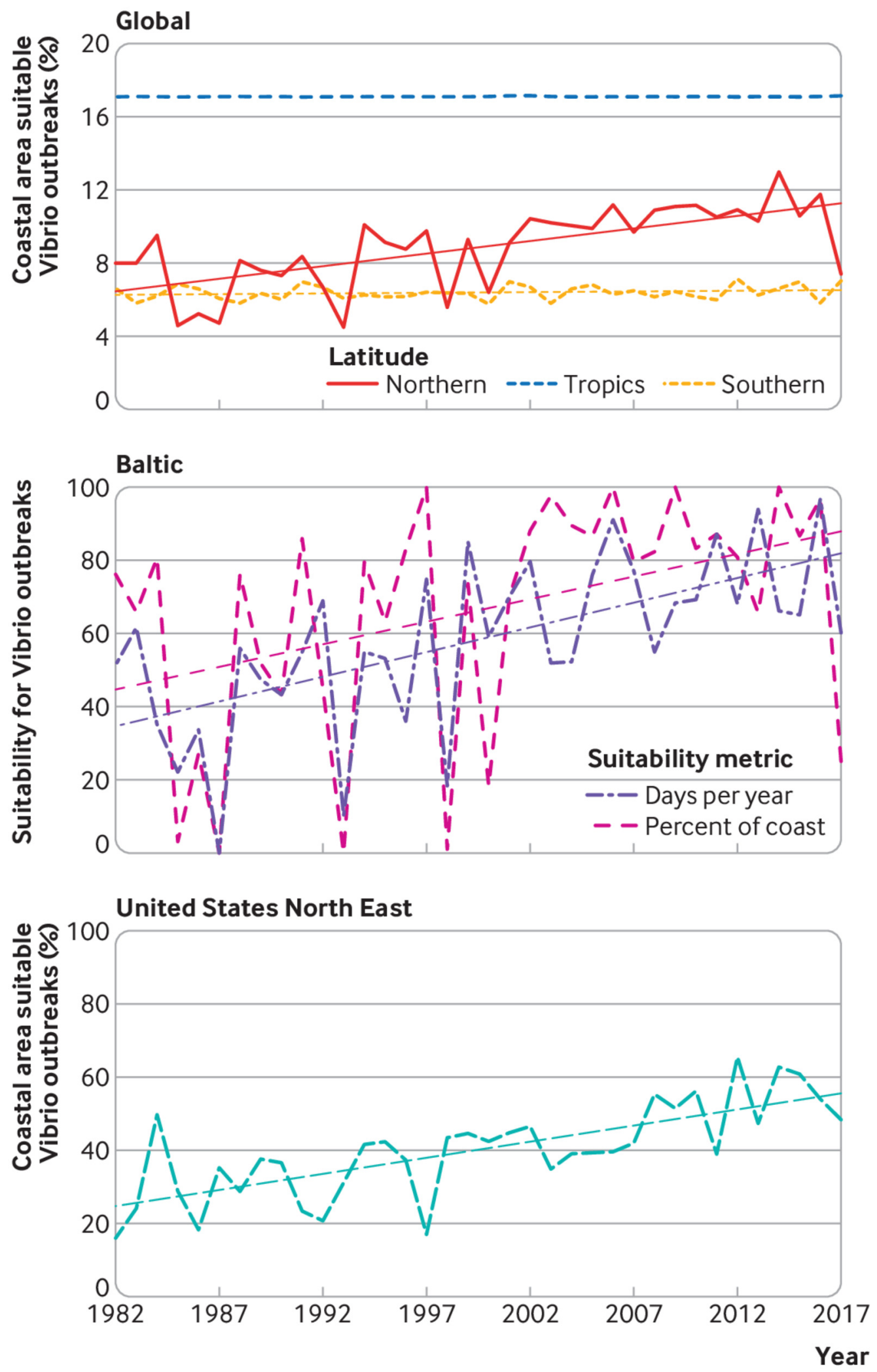

Fig 4 | Change in environmental suitability for pathogenic Vibrio outbreaks as determined by observed correlations with sea surface temperatures and ocean salinity. This model suggests that suitability is increasing predominantly in the northern hemisphere (top; northern latitudes $=40-70^{\circ} \mathrm{N}$; tropical latitudes $=25^{\circ} \mathrm{S}-40^{\circ} \mathrm{N}$; southern latitudes $=25-40^{\circ} \mathrm{S}$ ). More detailed analysis by regions shows, for example, that the Baltic Sea (middle) and the United States north east coast (bottom) are also increasingly suitable for Vibrio outbreaks due to climate change. For details on methodology and expanded interpretations, see the Lancet Countdown on Health and Climate Change ${ }^{19}$ ). 


\section{Challenges}

Each CSID indicator aims to capture environmental suitability of disease transmission by mathematically linking preferred conditions for transmission with climate input data. This allows the long term assessment of how environmental suitability for disease transmission has changed in recent decades, providing an initial step towards the attribution of disease risk to anthropogenic climate change.

Attribution of the underlying climate trends to human greenhouse gas emissions is highly robust ${ }^{20}$; however, it remains difficult to isolate the specific fraction of observed cases of each disease to climate change at large spatial scales given the range of other environmental and socioeconomic covariates at play. These include health inequality (ie, potential for the population to be harmed by a disease due to differential access to healthcare), land use, biodiversity, urbanization, travel and tourism, and global trade. Many of these factors are themselves influenced by climate change and exhibit strong spatial and temporal heterogeneities, illustrating the depth of the complexity of resolving realized climate change impacts on CSID burdens at continental or global scales. ${ }^{21}$

In addition, this set of indicators comprises several different methods (eg, threshold based, mechanistic, correlative models), datasets (eg, baseline gridded climate data), metrics (eg, percentage change versus raw change in suitability, indices of environmental suitability versus specific metrics such as vectorial capacity), and temporal windows (eg, baseline period, length of time series).

Different methods reflect the project's participatory, in kind, reformulation approach. However, a more systematic effort is needed to prioritize formally and objectively which diseases should be tracked, to develop standardized methodologies across diseases when possible, and to link outcomes to trends in other sectors, such as food security and access to healthcare, for a range of downstream uses.

\section{Data sharing, use, and public health application}

A wide range of public health stakeholders, ranging from the Global Climate and Health Alliance to the International Council of Nurses and the Royal College of Physicians, are increasingly engaged in climate change as a health issue. These professionals depend on both the generation of new medical evidence to drive this agenda forward, and the presentation of evidence in a way that they readily understand and can amplify to help drive both mitigation (eg, actions to reduce greenhouse gas emissions of healthcare infrastructure and services) and adaptation strategies (eg, identifying CSID hotspots, designing surveillance networks, early warning systems) to prepare for the changing risks from CSIDs to reduce their impacts.

Improving access to robust climate change risk assessments for health exposures and outcomes allows users to explore and appreciate the spatial and temporal heterogeneities in climate-related health risks relevant to local and coordinated management. For instance, the Lancet Countdown CSID indicators can be explored through an online visualization platform ${ }^{18}$ to highlight geographic areas that may be experiencing increases or decreases in disease risk, identify locations that require more research for a more accurate understanding of CSID risk, or highlight human populations where inequity gaps require urgent intervention to reduce their vulnerability to emerging climate change related public health threats. Accessible data sharing platforms provide a powerful avenue for users to visualize and interact with data, to appreciate the current situation in the context of the longer term trajectory, and to evaluate the growing momentum of certain trends through time and the often invisible build-up towards potential health crises. They also highlight the potential downstream impacts that greenhouse gas emissions today could have on health outcomes in the future.

Furthermore, given the scale and pace of the challenge that climate change presents, CSID indicator outputs must be paired with dedicated efforts to ensure they are translated into languages and formats that a wide range of audiences understand, ideally co-designed with policy makers and potential users. The development of an extensive network of policy and research partners is necessary to link key health bodies (eg, World Health Assembly, the World Health Summit, and the United Nations Framework Convention on Climate Change's decision making body) with health scientists and practitioners. Similarly, scientific literature must be paired with policy briefings, engaging narrative, and creative outputs if it is to engage across disciplines and help to draw out the local media and policy stories that may otherwise be hidden (for examples, see Lancet Countdown ${ }^{22}$ ).

\section{Collaborations and investment}

Climate change is increasingly being recognized as a public health emergency. ${ }^{23}$ Health risks and impacts will continue to grow unless the global community raises its collective ambition to meet the Paris Agreement, which aims to keep the world below $2{ }^{\circ} \mathrm{C}$ warming, and preferably below $1.5^{\circ} \mathrm{C} .{ }^{24}$ This goal, however, requires

evidence-based, transformative, and immediate action to curb greenhouse gas emissions.

While monitoring changes in climate under the Paris Agreement is crucial, equally important is the monitoring of potential health risks related to climate change. Better data for tracking infectious disease in a warming world requires a robust evidence base, recogniing that the challenges posed by climate change to health are substantial in size, complexity, and scope.

Initiatives to track the impacts of climate change (including increased variability in extreme events) and the effects of adaptation efforts on CSIDs have recently emerged to meet this challenge. The development and implementation of indicators calls for international, multi-disciplinary research collaborations dedicated to monitoring, analysing, anticipating, and communicating the links between climate change and health across the world.

Greater investment is required to help such initiatives realize their full potential to accurately identify the contribution of climatic drivers of infectious disease risk across space and time. In turn, identification and dissemination of climate-disease trends will signpost researchers, policy makers, health professionals, and the general public towards more informed, pre-emptive mitigation and adaptation actions to guide public health practice to an accelerated response to what has been termed by WHO as the "greatest global health threat of the 21st century."7

\section{Key recommendations}

- Development, standardization, and implementation of climate change and health indicators requires multidisciplinary research collaborations and major investment

- A systematic assessment of climate sensitive infectious diseases is required to prioritize diseases for tracking

- Standardized methodologies across diseases are needed with outcomes linked to trends in other sectors 
- Indicator outputs should be accessible and translated into languages and formats for diverse audiences, co-designed with policy makers and users

- Scientific reports should be paired with policy briefings, engaging narrative, and creative outputs to maximize media coverage and policy engagement

Competing interests: We have read and understood BMJ policy on competing interests and have no interests to declare.

Provenance and peer review: Commissioned; externally peer reviewed.

This article is part of a series commissioned by The BMJ for the World Innovation Summit for Health (WISH) 2020. The BMJpeer reviewed, edited, and made the decisions to publish. The series, including open access fees, is funded by WISH.

1 Haines A. Global warming and health. BMJ1991;302:669-70.

doi: 10.1136/bmj.302.6778.669 pmid: 1867673

2 Climate Change: The IPCC 1990 and 1992 Assessments - IPCC. https://www.ipcc.ch/report/climate-change-the-ipcc-1990-and-1992-assessments/

3 Shope R. Global climate change and infectious diseases. Environ Health Perspect 1991;96:171-4. doi: 10.1289/ehp.9196171 pmid: 1820262

42018 fourth warmest year in continued warming trend, according to NASA, NOAA. Clim. Change Vital Signs Planet. https://climate.nasa.gov/news/2841/2018-fourth-warmest-year-in-continuedwarming-trend-according-to-nasa-noaa

5 McIntyre KM, Setzkorn C, Hepworth PJ, Morand S, Morse AP, Baylis M. Systematic assessment of the climate sensitivity of important human and domestic animals pathogens in Europe. SCi Rep 2017;7:7134. doi: 10.1038/s41598-017-06948-9 pmid: 28769039

6 Semenza JC, Rocklöv J, Penttinen P, Lindgren E. Observed and projected drivers of emerging infectious diseases in Europe. Ann N Y Acad Sci2016;1382:73-83. doi: 10.1111/nyas.13132 pmid: 27434370

7 WHO. WHO calls for urgent action to protect health from climate change - sign the call. WHO. https://www.who.int/globalchange/global-campaign/cop21/en/

8 Griggs D, Stafford-Smith M, Gaffney 0, etal. Policy: Sustainable development goals for people and planet. Nature 2013;495:305-7. doi: 10.1038/495305a pmid: 23518546

9 Jamison DT, Summers LH, Alleyne G, etal. Global health 2035: a world converging within a generation. Lancet2013;382:1898-955. doi: 10.1016/S0140-6736(13)62105-4 pmid: 24309475

10 Ebi KL, Hasegawa T, Hayes K, etal. Health risks of warming of $1.5^{\circ} \mathrm{C}, 2^{\circ} \mathrm{C}$, and higher, above pre-industrial temperatures. Environ Res Lett2018;13:063007doi: 10.1088/1748-9326/aac4bd.

11 Institute for Health Metrics and Evaluation (IHME). Global Burden of Disease. GBD, 2019.http://www.healthdata.org/gbd

12 English PB, Sinclair AH, Ross Z, etal. Environmental health indicators of climate change for the United States: findings from the State Environmental Health Indicator Collaborative. Environ Health Perspect 2009;117:1673-81. doi: 10.1289/ehp.0900708 pmid: 20049116

13 Centers for Disease Control and Prevention (CDC). Climate Change Indicators. 2019.https://ephtracking.cdc.gov/showClimateChangelndicators

14 Climate Change Indicators in the United States US EPA. https:/www.epa.gov/climate-indicators

15 Indicators. Eur. Environ. Agency. https://www.eea.europa.eu/data-and-maps/indicators

16 Wellcome Trust. Tracking global progress on climate change. 2019.https://wellcome.ac.uk/news/tracking-global-progress-climate-change

17 The Inter-Sectoral Impact Model Intercomparison Project. ISIMIP. https://www.isimip.org/protocol/\#isimip2b

18 Data Platform. Lancet Countdown. https://www.lancetcountdown.org/data-platform/

19 Watts N, Amann M, Arnell N, etal. The 2019 report of The Lancet Countdown on health and climate change: ensuring that the health of a child born today is not defined by a changing climate. Lancet 2019;394:1836-78. doi: 10.1016/S0140-6736(19)32596-6 pmid: 31733928

20 IPCC. Climate Change 2013: The Physical Science Basis. Contribution of Working Group I to the Fifth Assessment Report of the Intergovernmental Panel on Climate Change. Cambridge University Press, 2013.

21 Semenza JC, Lindgren E, Balkanyi L, etal. Determinants and drivers of infectious disease threat events in Europe. Emerg Infect Dis 2016;22:581-9. doi: 10.3201/eid2204.151073 pmid: 26982104

22 Resources. Lancet Countdown. https://www.lancetcountdown.org/resources/

23 Harmer A, Eder B, Gepp S, Leetz A, van de Pas R. WHO should declare climate change a public health emergency. BMJ2020;368:m797. doi: 10.1136/bmj.m797 pmid: 32229481

24 Global Warming of $1.5^{\circ} \mathrm{C}-$. https://www.ipcc.ch/sr15/

This is an Open Access article distributed in accordance with the Creative Commons Attribution Non Commercial (CC BY-NC 4.0) license, which permits others to distribute, remix, adapt, build upon this work non-commercially, and license their derivative works on different terms, provided the original work is properly cited and the use is non-commercial. See: http://creativecommons.org/licenses/bync/4.0/. 